\title{
Time Comparison of Intensive Care Units With and Without Digital Viewing Systems
}

\author{
Louis M. Humphrey, Kevin Fitzpatrick, Natasha Atallah, and Carl E. Ravin
}

\begin{abstract}
As hospital radiology departments and intensive care units (ICUs) make plans to use or expand the usage of digital data outside of the radiology department, the need to assess the requirements of the potential recipients in the ICUs has become more important. The present operations in an ICU that uses digital viewing instead of film has been compared with a unit that does not. The difference in time between $x$-ray exposure and final image viewing was determined in both settings and compared. In this preliminary study, significant differences were found between the two units. The odds of having examination results actually accessed by the ordering physician in an hour or less were 9.5 times greater for the unit with digital viewing capability than for the one without it.

Copyright $\mathbb{1} 1993$ by W.B. Saunders Company
\end{abstract}

KEY WORDS: intensive care units (ICUs), digital radiography, picture archival and communications system (PACS), image transmission.

$\mathbf{I}$ T HAS BEEN SHOWN that rapid access to radiographic images and other radiology data can have a significant impact on patient stay in an intensive care unit (ICU) environment. ${ }^{1}$ The effect of image transmission to ICUs has been studied for several years. Several different schemes for controlling and transmitting data have been used. In many cases, the images sent to the ICU were images digitized from conventional film by a laser digitizer. ${ }^{1-4}$ These systems require human intervention to insure that the images were both digitized and transmitted. Other systems using computed radiography (CR) systems are more automaticoften requiring little or no additional effort to transmit the images. 5,6 The common feature in these systems is the ability to get information out of the radiology department to an ICU (or other environment) for review by the clinician. This information, supplied to the clinician, is important for patient care.

The application using CR combined with image transmission has proven to be an especially powerful combination. A CR system provides the image-to-image consistency needed in the ICU environment, whereas the image transmission capability of the picture archiving and communication system (PACS) provides the examination results to the clinician in a timely fashion.

An important consideration in implementing a system such as this was the workload it imposed on the users, both in radiology and the ICU. It was considered important to produce only small perturbations in the normal work flow in radiology and easy access to the images in the ICU. The CR-PACS combination has provided this flexibility.

\section{MATERIALS AND METHODS}

One of the important considerations in designing this study was determining which times were best to insure a need for imaging results within a reasonable time after an examination was ordered. It was determined that the "routine portable exam" film for rounds should be avoided. Most such examinations were performed between midnight and $8 \mathrm{am}$. Because the results were less urgent for these cases, those examinations in the midnight to 8 am period were excluded from the study. The hours between $4 \mathrm{pm}$ and midnight were selected for evaluation because the examinations ordered during this time frame were most likely to be in response to an urgent clinical need (new admissions, postoperative evaluations/instabilities, etc). The results of these examinations were usually needed as soon as possible by the clinician. This also represents the time the radiology department is in transition from the fully operational daytime environment to the decreased staffing levels of the nighttime environment.

Transmission of images from the radiology department to ICUs has been used for several years. Both the cardiac care unit (CCU) and the surgical ICU (SICU) have been receiving images automatically. In these cases, all images needed by the unit are transmitted, either through direct digital acquisition (The Philips Computed Radiography System (PCR), Philips Medical Systems, Shelton, CT) and transmission (CommView, Philips Medical Systems) or standard film digitization and transmission. A schematic diagram for this system is shown in Fig 1 . In most cases, the transmission of the images to the ICUs required minimal additional work by the technologist or clerk when digital radiography was used as the imaging modality. Images not acquired digitally were digitized and transmitted to the unit. Normal operations on both of these units included the use

From the Radiology Department and the Department of Surgen at the Duke University Medical Center, Durham, NC.

Address reprint requests to Louis $M$. Humphrey, $M A$, Department of Radiology, Duke University Medical Center, Enwin Road, PO Box 3808, Durham, NC 27710.

Copyright $\odot 1993$ by W.B. Saunders Company

0897-1889/93/0601-0004\$03.00/0 


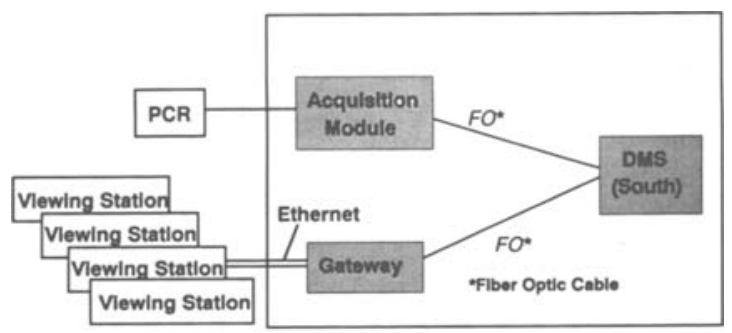

Fig 1. Digital imaging and transmission system.

of the PCR and CommView (PACS) for all portable examinations. Important images from other exams (CT, magnetic resonance etc) were digitized and transmitted when requested.

In the two units that are using a system for displaying images, SICU and CCU, very different patterns of usage have been observed. Based on this information, the next units to be installed were studied before installation so that the effect the system would have on the users could be determined and the impact on patient care could be assessed.

Each unit in the hospital uses radiology data differently. As a result, it has become important to understand how the image data sent to the unit will help treat the patients there. This time study is one of many steps needed to help understand how radiology data is used outside the radiology department.

The two units examined in this study were the SICU and the medical ICU (MICU). A digital viewing station is now in use by clinicians in the SICU, whereas the MICU clinicians have no definitive means of getting radiology data quickly except by either traveling to the radiology department to view the film or telephoning a radiologist for an interim verbal report. The usual method of communication had been the telephone. In such cases, the radiologist usually returned the call to the ordering physician when the interpretation was completed to relay any abnormal results and discuss the case before a treatment was begun. For those cases in which it was necessary to get the information faster, someone from the MICU would accompany the technologist back to the radiology department and look at the film as soon as it was processed. In such situations, the clinician was usually looking for significant abnormalities that had to be treated immediately.

In contrast, the SICU used a digital viewing system for all bedside work. Before the system was installed, copies of all portable films were sent manually to the unit. In both cases (film copies and digital viewing) images on the SICU were available for review for as long as the patient was there. With the digital system, review of images only required walking to a central nursing station and selecting the desired images from a list of patients on the unit. Immediate cases were usually available at the station in approximately the same time it took to process film for review by the radiologist. In addition, viewing images has become a part of the daily functions on the unit.

Both units were observed for 6 nights. All examinations that were ordered were timed beginning with the $x$-ray exposure and ending when the examination information was communicated to the ordering physician.
For the MICU study, the time measured included the time it took for the technologist to travel from the ICU after the exposure (to develop the film and for the radiologist to interpret it) as well as the time the radiologist spent waiting to get in touch with the clinician after the interpretation. For those cases in which the clinician went to the radiology department to view the film, the clock was stopped when the film was viewed. The time spent determining the action to take after the appropriate information had been communicated was not included in the study.

The periods used for the SICU were slightly different because of the presence of the viewing station. There, the period started when the exposure was made and ended when the ordering physician reviewed the examination at the viewing station or was told of the results by someone who had. In these cases, the radiologist was rarely involved in the process, although he/she was still expected to provide the official interpretation and alert the ordering physician whenever there were findings that should be considered when treating the patients. The radiologist was also available for consultation whenever the ordering physician had questions about an examination.

Reasons for ordering the examinations and the types of examinations ordered were not considered during the study. All examination results were considered to be needed as soon as possible.

The process followed from the ordering of the examination to the transmission of the results for the two units is shown in Figs $2 a$ and $2 b$. After the order was verified, the requisition was printed in the radiology department. In some cases, the unit would call the department to expedite the process. The technologist would then go to the unit and perform the examination and bring the exposed film back to the radiology department for processing and interpretation. For the SICU, the processing also resulted in the transmission of the image to the viewing station on the unit for use as needed. Simultaneously, the image produced was available to the radiologist for interpretation. For the MICU, the film produced was given to the radiologist for interpretation. If. the ordering physician requested immediate contact, the radiologist would usually call him/her and discuss the result. If not, the radiologist would write a preliminary result on a form available to the clinician, who then had the responsibility to retrieve it. In some cases, a representative from the floor would come to the department to review the film as it emerged from the processor and return to the unit without consultation. In other cases, the representative would consult with the radiologist in addition to viewing the film.

All observations during this study were made between August 12, 1991 and August 24, 1991 and timed with a stopwatch by an observer. The ordering physicians were not told that they were being observed, although the radiology department and some unit personnel were made aware of the study to help insure that the times measured were accurate and the results from the examinations ordered were needed quickly.

\section{RESULTS}

The mean time between $x$-ray exposure for an ordered examination and the requesting physi- 


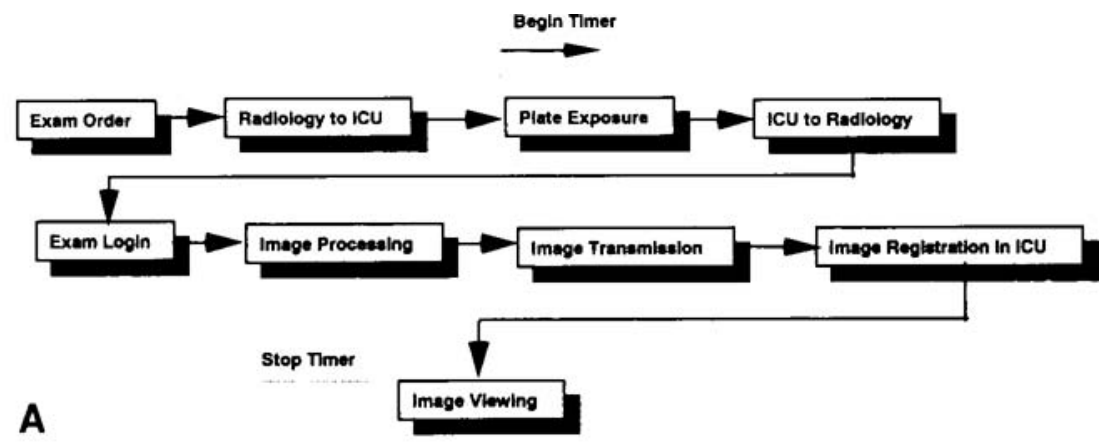

Fig 2. (A) SICU examination process. (B) MICU examination process.

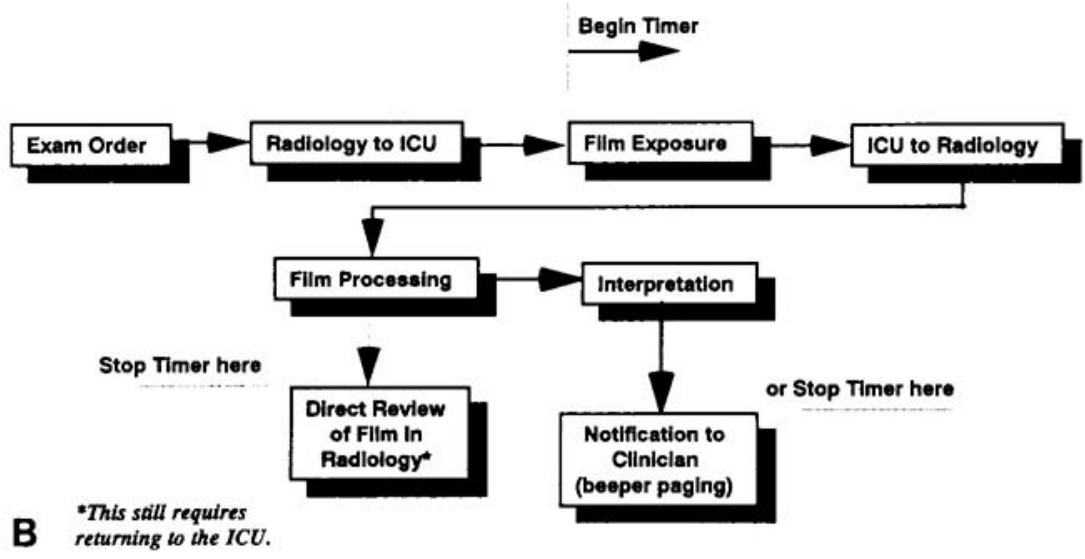

cian reviewing the study or obtaining the results was approximately 39 minutes for the SICU and 78 minutes for the MICU. The longest interval for the SICU was approximately 84 minutes, whereas that for the MICU was nearly 2.5 hours (see Fig 3). As part of bracketing the time within which the studied radiology examination results are useful in intensive care settings, it was determined that for immediate examinations, 1 hour was close to the maximum time between the determination by the clinician that there is a need to perform the requested examination and subsequent return of examination information to that clinician for analysis and integration. Further discussions indicated that the "clock" does not really start for the clinician

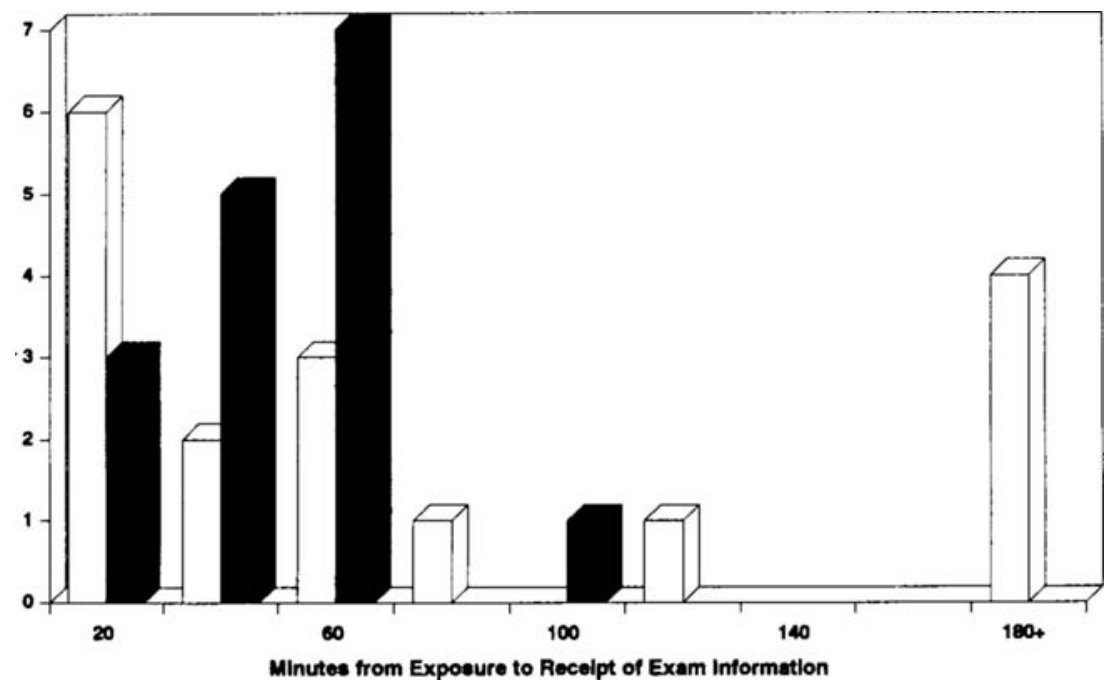

Fig 3. Plot of times the examination information was returned to clinicians within a measured time (20 min intervals). $\square$, MICU; 口, sICU. 
until the radiology technologist arrives on the unit to perform the examination. Results returned after that time tended to have little or no part in determining the next course of treatment for the patient. Consequently, when the interval from exposure to notification was divided into two groups-less than or equal to 1 hour and greater than 1 hour-(This interval was chosen before any of the exam data was analyzed to avoid any prejudicial slant to the findings.) - there was a significant difference in the overall (perceived and actual) availability of radiology information to the clinicians on the two units. When a odds ratio test was applied $(P=.0425)$, it was determined that clinicians on the SICU were 9.5 times more likely to access the results of an ordered examination within 1 hour after an examination was performed than were the clinicians on the MICU.

The interval data for the two units was not normally distributed. Roughly one third of the MICU cases were read and communicated to the clinician within the first 20 minutes. These were interpreted as the cases for which results were needed immediately, or in a time shorter than the median time between exposure and notification $(57.5 \mathrm{~min})$. Most of these cases involved sending a person back with the technologist to look at the examination as soon as it was processed. The remainder are scattered over a 2.5-hour interval. In contrast, the observations in the SICU show that the examination information was viewed more evenly over a period of approximately 60 minutes, with a single point at approximately 84 minutes. The clinicians in the MICU-using conventional film methodswere given access to the examination results within 1 hour $60 \%$ of the time, while the SICU clinicians-using a digital viewing stationviewed the examinations $94 \%$ of the time within that same period.

\section{DISCUSSION}

A preliminary study of two ICUs at Duke University (Durham, NC) has shown that the unit with digital viewing capability (the SICU) is much more likely ( 9.5 times) to review radiology information for patient care decisions within 1 hour than the unit that does not have the system (the MICU). While the MICU is able to review the radiology data in the most critical cases within 20 minutes very effectively, the remaining examinations tend to be reviewed over a longer period. The effort involved in reviewing images on the MICU is significantly greater than that on the SICU. As a result, only the most pressing and questionable cases are rigorously pursued by going to the radiology department with the technologist immediately after the exposure or by calling to speak to a radiologist, etc. Furthermore, the involved radiologist must be informed of the urgency of the case and make a sustained effort to communicate the results to the clinician. By comparison, the SICU is less dependent on people to provide the information to the clinician in these cases. Nor is the clinician or the radiologist required to make a sustained effort to be sure the radiology data (images and interpretation) is returned to the correct person. Within a reasonable period after the examination is performed (usually no more than 10 minutes including travel time), the image is available for review on monitors in the unit. The clinician is free to review the image anytime after that, and the radiologist is always available for consultation.

The digital viewing station in the SICU has enabled the clinician to review the radiology data when needed for purposes related to patient care. Instead, both the clinician and the radiologist are free to handle other matters and respond to other pressing cases. The clinician is able to review the radiology data when needed for purposes related to patient care. The critical time between exposure and getting the results of the examination appears to be on the order of 1 hour. With this interval, the likelihood of the SICU retrieving the information as needed is 9.5 times higher than for the MICU. It must be emphasized that because the two units operate differently, it cannot be inferred from this preliminary study that all of the differences between the units is due to the presence of a digital viewing station on one and not on the other. A more detailed study of the same unit before and after installation of a viewing station is needed to truly assess the value of the viewing station additions. However, given the same limits within which the radiology data was considered important, this study is a good indicator of the potential of this technology for patient care. 
As new technology (begun in the radiology department) expands into other areas of the hospital, studies of this kind are important for assessing the impact of the technology on these areas. These studies help to show the needs of the other areas in the hospital and their dependence on the radiology department's data for decisions regarding patient care. The critical connection between the ordering physician and the radiologist relies on people for conveying results. This link, though strong, is often timeconsuming. When the need for information is great, it is not an optimal link. An automated system provides a reliable connection so that data produced in the radiology department is available quickly to the requesting physician.

\section{REFERENCES}

1. Arenson RL, Seshadri SB, Kundel HL, et al: Clinical evaluation of a medical image management system for chest images. AJR Am J Roentgenol 150:55-59, 1988

2. Franken EA Jr, Smith WL, Berbaum KS, et al: Comparison of a PACS workstation with conventional film for interpretation of neonatal examinations: A paired comparison study. Pediatr Radiol 21:336-340, 1991

3. DeSimone D, Kundel HL, Arenson RL, et al: Effect of a digital imaging network on physician behavior in an intensive care unit. Radiology 169:41-44, 1988
4. Gillespy T, Staab EV, Lawrence E: Electronic imaging in a teaching hospital intensive care unit: Evaluation of the clinical review system. J Dig Imag 3:124-128, 1990

5. Ravin CE: Initial experience with automatic image transmission to an intensive care unit using Picture Archiving and Communications System technology. J Dig Imag 3:195-199, 1990

6. Witt RM, Cohen MD, Appledorn CR: Initial experience with a radiology imaging network to newborn and intensive care units. J Dig Imag 4:39-42, 1991 